\title{
Symbol error rate analysis of DMIMO antenna systems in linear cell Rongrong $\mathrm{Xu}^{1, a}$, Yueheng Li, Ping Huang, Meiyan Ju, Guoping Tan \\ ${ }^{1}$ College of Computer and Information, Hohai University, Nanjing, Jiangsu, 211100, China aemail:1059805281@qq.com
}

Key words: Linear cell, Distributed MIMO systems, Symbol error rate, Composite channel

\begin{abstract}
In this paper, the symbol error rate of distributed MIMO antenna systems in a linear cell is analyzed. Firstly, a composite channel model which includes the fast fading, shadow fading and path loss is established. Then, a closed form expression of symbol error rate for point-to-point downlink MIMO communication system under higher signal-to-noise ratio (SNR) is deduced. Next, by considering the nonuniform distribution of a mobile station, the closed form expression of average symbol error rate in the whole cell is further derived. Numerical results show that the theoretical expressions derived in this paper can match the actual system performance well.
\end{abstract}

\section{Introduction}

With the rapid development of wireless communication technology, large capacity, high speed and high quality data transmission have become the systematic design targets of a wireless communication system. Distributed MIMO (DMIMO) system with its significant performance advantages has been widely concentrated by many researchers in recent years. High quality data transmission as well as the system error rate plays an important role in the whole system's performance.

Researches about DMIMO systems have achieved many results. The performance of a new MIMO system and a new space time block code design under the fast fading and slow fading channels were studied in [1]. The outage performance of MIMO system which includes large-scale fading and small-scale fading was researched [2], and the results showed that the system parameters had effect on the system performance. However, these documents did not analyze the symbol error rate of DMIMO systems in a linear cell.

This paper will focus on the system error rate of DMIMO systems in a linear cell. Assuming that the composite fading channel includes the fast fading, shadow fading and path loss, and that the noise interference from other linear cells can be ignored and the transmitter adopts orthogonal space-time block coding (OSTBC), the closed form expressions of the point-to-point downlink symbol error rate and the average symbol error rate are deduced.

\section{System Model}

The structure of a basic DMIMO system in a linear cell is shown in Fig 1, which consists of $N$ distributed antenna ports (APs) and one mobile station (MS). All $A P_{n}(n=1,2, \cdots N)$, each of which is equipped with one transmit antenna, are distributed on the same line and connected with the central processor through a wired link. The MS with $L$ receive antennas is on the same line that APs are distributed on.

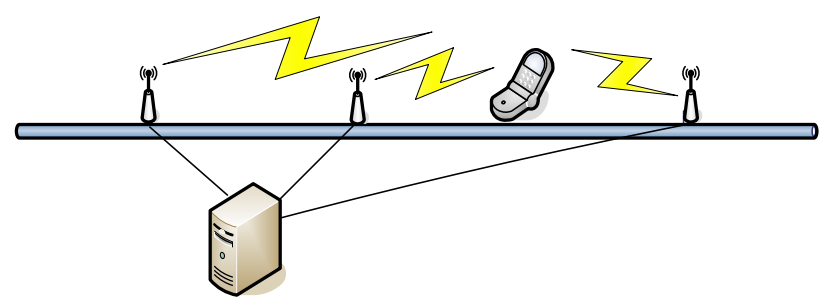

Fig.1. Illustration of a linear DMIMO system 
In this paper, we only study the downlink transmission of the linear DMIMO system. The information data sent by the base station is encoded by OSTBC, which generates $N$ parallel data sequences. The parallel data sequences $\left(x_{t}^{1}, x_{t}^{2}, \cdots, x_{t}^{N}\right)$ are transmitted by $N$ antenna ports simultaneously at each time instant $t$. Assuming that the composite fading channel is quasi-static, the signal in receive antennas at the same time instant can be expressed as

$$
\mathbf{Y}_{\mathbf{t}}=\sqrt{E_{S}} \mathbf{H} \mathbf{X}_{\mathbf{t}}+\mathbf{N}_{\mathbf{0}}
$$

Where $E_{S}$ is the transmit power; $\mathbf{X}_{\mathbf{t}}=\left[x_{t}^{1}, x_{t}^{2} \cdots, x_{t}^{N}\right]^{\mathrm{T}}$ is the transmitted symbol vector at time instant $t ; \mathbf{N}_{0}$ is the noise vector, whose elements are additive white Gaussian noise (AWGN) random variables with zero mean and same variance $\sigma^{2} ; \mathbf{H}$ is an $L \times N$ matrix, which represents the fading between the $N$ transmit antennas and $L$ receive antennas, and its elements are given by

$$
[\mathbf{H}]_{l, n}=g_{l, n} \sqrt{\zeta_{n}},(l=1,2, \cdots L ; n=1,2, \cdots N)
$$

Where $[\mathbf{H}]_{l, n}$ is the element of matrix $\mathbf{H}$, which represents the fading coefficient between the $l$ th receive antenna and $A P_{n} ; g_{l, n}$ and $\zeta_{n}$ represent the small-scale fading and large-scale fading between the $l$ th receive antenna and $A P_{n}$ respectively; $g_{l, n}$ can be modeled as a complex Gauss random variable with zero mean and unit variance; $\zeta_{n}$ is the log-normal distribution with mean $\mu_{n}$ and variance $\sigma_{n}{ }^{2}\left(10 \log _{10} \zeta_{n} \sim\left(\mu_{n}, \sigma_{n}^{2}\right)\right)$. The path loss $\mu_{n}$ between MS and $A P_{n}$ is expressed as

$$
\mu_{n}=10 \lg \left[\eta\left(\frac{d_{0}}{d}\right)^{\tau}\right]
$$

Where $\eta$ is the average fading constant; $d_{0}$ is the reference distance; $d$ represents the distance between the transmitter and receiver, and $\tau$ is path loss exponent.

\section{Analysis of Symbol Error Rate}

As shown in Fig.2, the MS is located in a fixed position in the cell, and $d_{n}=\left|r-r_{n}\right|(n=1,2, \cdots, N)$ represents the distance between the $A P_{n}$ and mobile station.

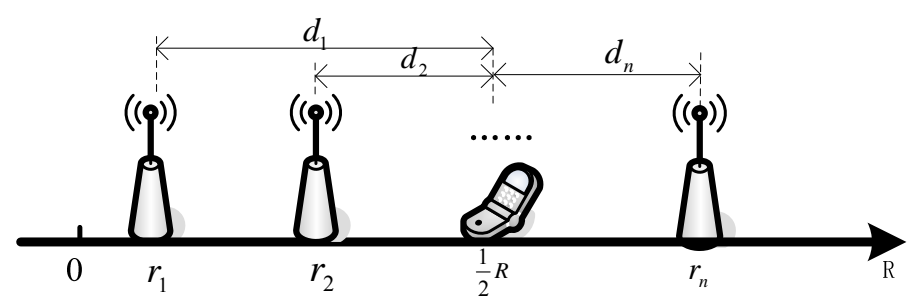

Fig.2.Illustration of a linear DMIMO system when position of MS is fixed

Assuming that the receiver adopts maximum likelihood (ML) algorithm to estimate the received signal, the estimation criterion can be expressed as

$$
\min \left\{\sum_{l=1}^{L} \sum_{t=1}^{T}\left|y_{t}-\sqrt{E_{s}} \sum_{n=1}^{N} g_{l, n} \sqrt{\zeta_{n}} x_{n}^{t}\right|^{2}\right\}
$$

According to [6], the decoding result of the OSTBC is: 


$$
\widehat{c_{n}}=\sum_{l=1}^{L} \sum_{n=1}^{N}\left(\left|g_{l, n}\right|^{2} \zeta_{n}\right) c_{n}+\hat{z}, n=1, \cdots, N
$$

Where $c_{n}$ is a symbol which needs to be encoded; $\hat{z}$ is AWGN with zero mean and variance $\left(\sum_{l=1}^{L} \sum_{n=1}^{N}\left|g_{l, n}\right|^{2} \zeta_{n}\right) \sigma^{2}$

According to [4], the expression of conditional symbol error rate (SER) is derived under the MPSK (M-ary phase shift keying) modulation:

$$
P_{S E R}\left(E /\left\{\gamma_{n}\right\}_{n=1}^{N}\right)=\frac{1}{\pi} \int_{0}^{(M-1) \pi / M} \exp \left(-\frac{E_{s}}{N_{0}} \frac{g_{P S K}}{\sin ^{2} \phi}\right) d \phi=\frac{1}{\pi} \int_{0}^{(M-1) \pi / M} \prod_{n=1}^{N} \exp \left(-\frac{g_{P S K} \gamma_{n}}{\sin ^{2} \phi}\right) d \phi
$$

In above formula, $g_{P S K}=\sin ^{2}(\pi / M)$.

We operate the statistical average over $\gamma_{n}$ in (6), resulting in ${ }^{[4]}$ :

$$
P_{S E R} \mid\left\{\zeta_{n}\right\}_{1}^{N}=\frac{1}{\pi} \int_{0}^{\frac{(M-1) \pi}{M}} \prod_{n=1}^{N}\left[1+\frac{\bar{\gamma}_{n} \sin ^{2}(\pi / M)}{\mathrm{m} \sin ^{2} \phi}\right]^{-L m} d \phi
$$

Where $P_{\mathrm{SEP}} \mid\left\{\zeta_{n}\right\}_{1}^{N}$ is SER based on the large-scale fading; $m$ is the shape factor in Nakagami fading channels; $\gamma_{n}$ and $\bar{\gamma}_{n}$ are instantaneous and average SNRs at the receiver respectively. $\gamma_{n}$ and $\overline{\gamma_{n}}$ can be expressed as

$$
\begin{aligned}
& \gamma_{n}=\frac{E_{s}\left|g_{l, n}\right|^{2} \zeta_{n}}{\sigma^{2}} \\
& \overline{\gamma_{n}}=\frac{E_{s} \zeta_{n}}{\sigma^{2}}
\end{aligned}
$$

With the condition of higher SNR, (7) can be approximately written in the following form

$$
P_{\mathrm{SER}} \mid\left\{\zeta_{n}\right\}_{1}^{N} \approx \frac{1}{\pi}\left[\prod_{n=1}^{N} \overline{\gamma_{n}} \frac{\left(\sin ^{2}(\pi / M)\right)}{m}\right]^{-L m} \int_{0}^{\frac{(M-1) \pi}{M}} \sin ^{2 N L m} \phi d \phi
$$

Because $\sin ^{2 K} \theta$ can be expressed as

$$
\sin ^{2 K} \theta=2^{-2 K}\left[\left(\begin{array}{c}
2 K \\
K
\end{array}\right)+2 \sum_{\eta=0}^{K-1}\left(\begin{array}{c}
2 K \\
\eta
\end{array}\right)(-1)^{K-\eta} \cos [2(K-\eta) \theta]\right]
$$

the integral $\int_{0}^{\frac{(M-1) \pi}{M}} \sin ^{2 N L m} \phi d \phi$ in (10) can be given as

$$
\int_{0}^{\frac{(M-1) \pi}{M}} \sin ^{2 N L m} \phi d \phi=2^{-2 N L m}\left[\left(\begin{array}{c}
2 N L m \\
N L m
\end{array}\right) \frac{(M-1) \pi}{M}-\sum_{\eta=0}^{N L m-1}\left(\begin{array}{c}
2 N L m \\
\eta
\end{array}\right)(-1)^{N L m-\eta} \frac{\sin \frac{2(N L m-\eta) \pi}{M}}{N L m-\eta}\right]
$$

Substituting the following formula into $\psi$ 


$$
\psi=\frac{1}{[2 \sin (\pi / M)]^{2 N L m}}\left[\left(\begin{array}{c}
2 N L m \\
N L m
\end{array}\right) \frac{M-1}{M}-\sum_{n=1}^{N L m}\left(\begin{array}{c}
2 N L m \\
N L m-n
\end{array}\right)(-1)^{n} \frac{\sin (2 \pi n / M)}{\pi n}\right]
$$

Where $n=N L m-\eta$, then (10) can be written in the following form

$$
P_{S E R} \mid\left\{\zeta_{n}\right\}_{1}^{N}=\psi\left(\prod_{n=1}^{N} \overline{\gamma_{n}}\right)^{-L m}=\psi\left(\prod_{n=1}^{N} \frac{E_{s}}{\sigma^{2}} \zeta_{n}\right)^{-L m}=\psi\left(\frac{E_{s}}{\sigma^{2}}\right)^{-N L m}\left(\prod_{n=1}^{N} \frac{1}{\zeta_{n}}\right)^{L m}
$$

Since $\zeta_{n}$ is log-normal distributed, $\prod_{n=1}^{N_{t}} \frac{1}{\zeta_{n}}$ is also log-normal distribution, that is, $10 \lg \left(\prod_{n=1}^{N} \frac{1}{\zeta_{n}}\right) \sim N\left(\tilde{u}, \tilde{\sigma}^{2}\right)$. Here $\tilde{u}$ and $\tilde{\sigma}^{2}$ are given as

$$
\left\{\begin{array}{l}
\tilde{u}=-\sum_{n=1}^{N} \mu_{n} \\
\tilde{\sigma}^{2}=\sum_{n=1}^{N} \sigma_{n}^{2}
\end{array}\right.
$$

We still operate statistical average over the large-scale fading random variable $\zeta_{n}$ in (14), resulting in

$$
P_{\mathrm{SER}}(r)=E_{\zeta_{1}, \cdots, \zeta_{n}}\left\{P_{\mathrm{SEP}} \mid\left\{\zeta_{n}\right\}_{1}^{N}\right\}=\psi\left(\frac{E_{s}}{\sigma^{2}}\right)^{-N L m} \exp \left(\frac{(L m)^{2}}{2 \xi^{2}} \tilde{\sigma}^{2}\right) \exp \left(-\frac{L m}{\xi} \sum_{n=1}^{N} \mu_{n}\right)
$$

Where $\xi=10 / \ln 10 ; \mu_{n}$ is the path loss between the mobile station and $A P_{n}$, which can be given by

$$
\mu_{n}=10 \lg \left[\left(\frac{d_{0}}{d_{n}}\right)^{\tau}\right]
$$

Here $d_{0}$ is reference distance; $d_{n}=\left|r-r_{n}\right|(n=1,2, \cdots, N)$ represents the distance between $A P_{n}$ and MS; $\tau$ is path loss exponent.

Using (17), (16) can be rewritten as

$$
P_{\mathrm{SER}}(r)=\psi\left(\frac{E_{s}}{\sigma^{2}}\right)^{-N L m} d_{0}^{-N m L \tau} \exp \left(\frac{(L m)^{2}}{2 \xi^{2}} \tilde{\sigma}^{2}\right)\left(\prod_{n=1}^{N} d_{n}^{L m \tau}\right)
$$

Until now, the closed form expression of symbol error rate of point to point link is deduced. Assuming that MS is nonuniform distribution, the linear cell can be divided into two parts. The first part $\psi 1$ is from the position 0 to the center of the cell $R_{h}$, and the second part $\psi 2$ is from the center $R_{h}$ to the position $R$. This model can be illustrated as in Fig.3.

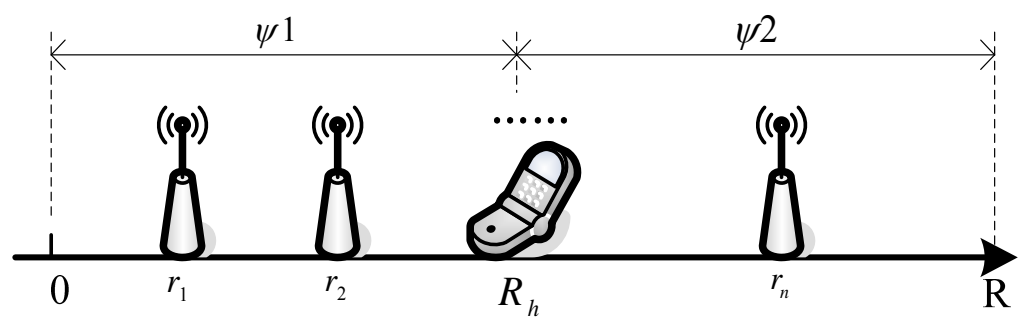

Fig.3. Illustration of a linear DMIMO system when MS is nonuniform distribution The probability density function (PDF) of the MS position is 


$$
f(r)=\left\{\begin{array}{cc}
\lambda / R & 0 \leq r \leq R_{h} \\
(1-\lambda) /\left(R-R_{h}\right) & R_{h} \leq r \leq R
\end{array}\right.
$$

Where $\lambda \in[0,1]$ represents the probability of MS distributed in section $\psi_{1}$; when $\lambda<R_{h} / R$, the distribution of MS is concentrated in $\psi_{1}$; when $\lambda>R_{h} / R$, the distribution of MS is concentrated in $\psi_{2}$. Especially when $\lambda=1$, the MS is concentrated in $\psi_{1}$; and when $\lambda=0$, MS is concentrated in $\psi_{2}$.

Operating the statistical average over $d_{n}$ in (18), we achieve

$$
\overline{P_{\mathrm{SER}}}(r)=\int_{0}^{R} P_{\mathrm{SER}}(r) f(r) d r=\psi\left(\frac{E_{s}}{\sigma_{z}{ }^{2}}\right)^{-N L m} d_{0}^{-N L \tau} \exp \left(\frac{(L m)^{2}}{2 \xi^{2}} \tilde{\sigma}^{2}\right) \int_{0}^{R}\left(\prod_{n=1}^{N} d_{n}^{L m \tau}\right) f(r) d r
$$

\section{Simulation Results and Analysis}

Simulation parameters used in the paper are summarized in Table 1.

Table 1 Simulation parameters

\begin{tabular}{cc}
\hline Simulation parameters & Value \\
\hline number of APs, N & 2 \\
number of receive antennas, $\mathrm{L}$ & 1 \\
length of the linear cell, $\mathrm{R}$ & $2 \mathrm{~km}$ \\
position of $A P_{1}, \mathrm{r} 1$ & $200 \mathrm{~m}$ \\
position of $A P_{2}, \mathrm{r} 2$ & $1800 \mathrm{~m}$ \\
Position of MS & $1000 \mathrm{~m}$ \\
standard deviation of shadow fading, $\sigma$ & $4 \mathrm{~dB}$ \\
path loss exponent, $\alpha$ & 4 \\
reference distance, $d_{0}$ & $40 \mathrm{~m}$ \\
MPSK, M & 2 \\
value of m in Nakagami fading & 1 \\
\hline
\end{tabular}

The results of simulations are shown in Fig.4 and Fig.5.
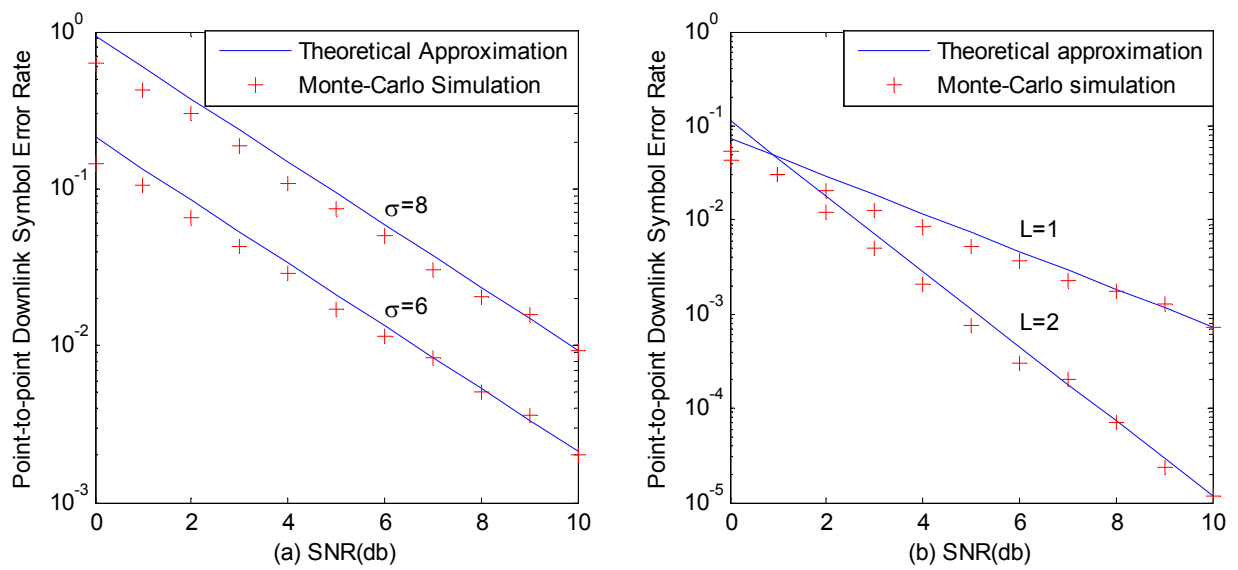

Fig.4. SER performances of DMIMO system when MS is fixed 

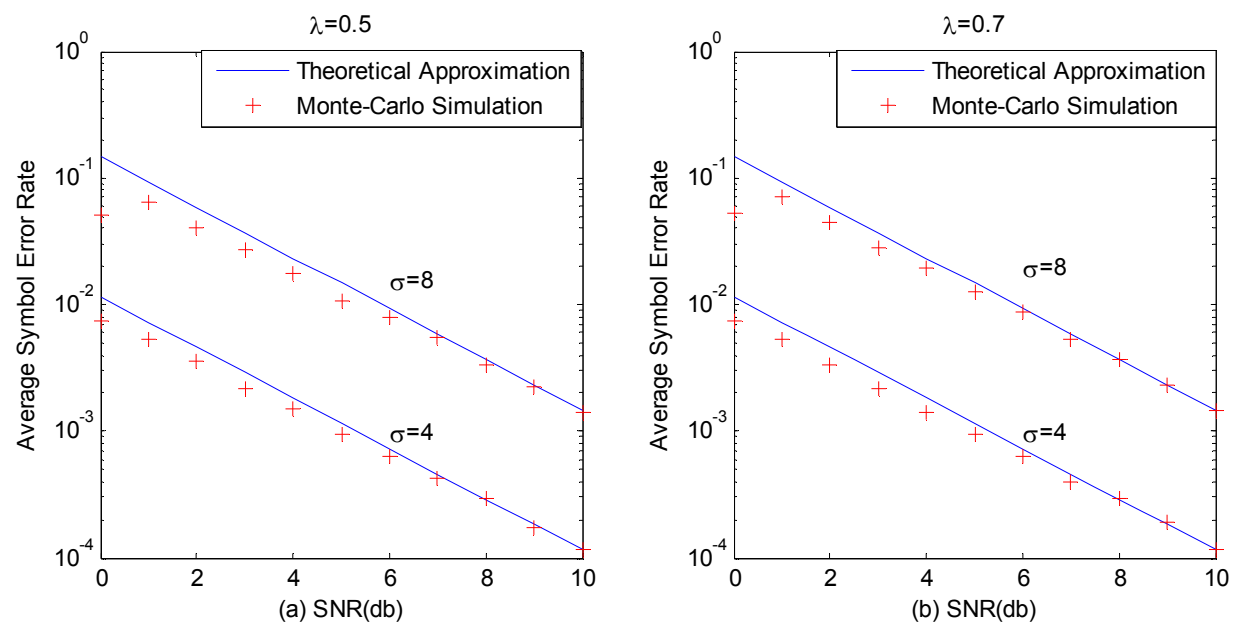

Fig.5. SER performances of DMIMO system when the position of MS is nonuniform distribution

In Fig.4, from the sub-graphs (a) and (b), we can see that while the standard deviation of shadow fading $\sigma$ is more greater, the point-to-point downlink symbol error rate is more larger; and when the number of receiver antennas $L$ is more greater, the symbol error rate is more smaller. From the sub-graphs (a) and (b) in Fig.5, we can find that whether the MS is uniform distribution or nonuniform distribution, the more standard deviation of shadow fading $\sigma$ is, the worse the system performance is.

\section{Conclusions}

In this paper, the symbol error rate of DMIMO systems in a linear cell is studied. Firstly, the expression of the signal at receiver is given and the maximum likelihood algorithm is adopted to estimate the received signal. Then the closed form expressions of the point-to-point downlink symbol error rate and the average symbol error rate are deduced. It is shown that the error rate formula can exactly reflect the actual DMIMO system performance, so the results of this paper can give constructive advice for the design of linear DMIMO systems.

\section{References}

[1] Yuan J, Chen Z, Vucetic B and Firmanto W. Performance and Design of Space-Time Coding in Fading Channels [J]. IEEE transactions On Communications, 2003, 51(12): 1991-1996.

[2] Roh W and Paulraj A. Outage Performance of the Distributed Antenna Systems in a Composite Fading Channel [J]. IEEE Veh. Technology Conf. (VTC'02 Fall), 2002, 3: 1520-1524.

[3] Gradshteyn IS and Ryzhik IM. Table of Integrals, Series and Products, 7th Edition [M]. San Diego: Academic Press, 2007.

[4] Simon MK and Alouini MS. Digital Communication over Fading Channels, 2nd ed. [M]. New York: Wiley, 2005.

[5] Stüber GL. Principles of mobile communication [M]. Springer, 2011.

[6] Larsson EG, Stoica P. Space-time block coding for wireless communications [M]. Cambridge university press, 2008 .

[7] Paulraj A, Nabar R, Gore D. Introduction to space-time wireless communications [M]. Cambridge university press, 2003. 